\title{
Disseminated nocardiosis with multisite involvement in an immunocompetent patient
}

\author{
Arjun Balachandar MD, Alice Zhabokritsky MD, Larissa M. Matukas MD
}

口 Cite as: CMAJ 2020 August 17;192:E956-9. doi: 10.1503/cmaj.200153

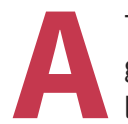

73-year-old retired engineer presented to the emergency department with a 4-month history of transient left arm and leg paresthesias lasting minutes. His medical history included glaucoma and a 20 pack-year history of smoking, and he used no medication other than eye drops. He had immigrated to Canada from India 50 years ago but had no recent travel history. As a result of his clinical presentation and a normal noncontrast computed tomography (CT) scan of the head, he was diagnosed with a transient ischemic attack and sent home. Outpatient follow-up, including a CT angiogram and magnetic resonance imaging (MRI) scan of the brain, was organized by his family physician.

During a month of waiting for investigations, the patient gradually developed a cough, exertional dyspnea and eventually hemoptysis, for which he did not seek medical attention. He also had night sweats without fever or chills and a $3 \mathrm{~kg}$ weight loss. The CT angiogram showed no intracranial abnor-

\section{KEY POINTS}

- Nocardia is found worldwide in soil and decaying plant matter and can cause infection through inhalation of cell fragments in dust particles.

- In patients presenting with a lung mass and associated brain lesions, the differential diagnosis should include infections like tuberculosis and disseminated nocardiosis.

- Nocardiosis most often occurs in immunocompromised hosts, but as many as one-third of affected patients are immunocompetent.

- High-dose trimethoprim-sulfamethoxazole is the initial treatment of choice for nocardiosis.

mality but partially captured his lung apices, revealing a right apical cavitating lesion with associated adenopathy. Within 1 week, his family physician had arranged for a CT scan of the
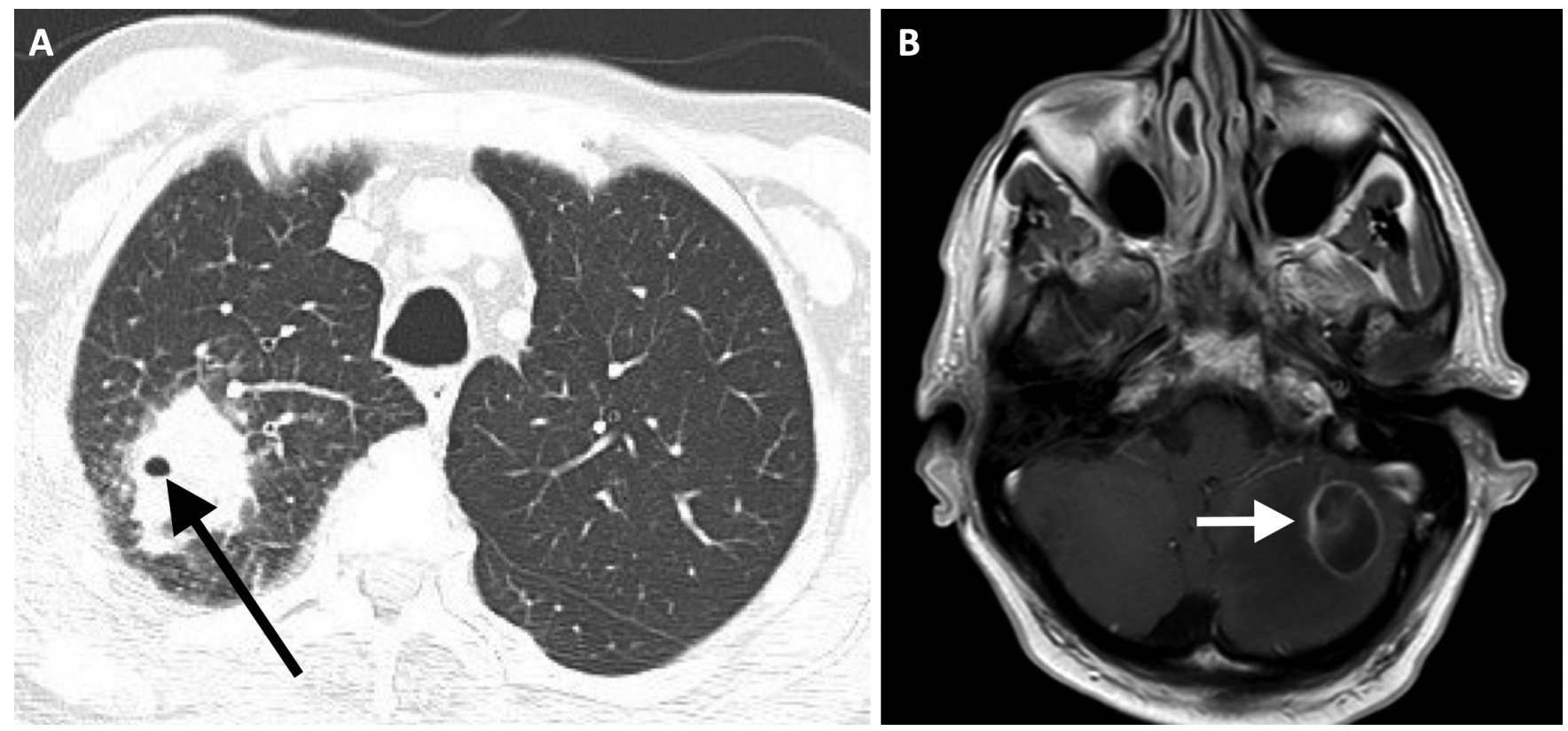

Figure 1: Imaging studies in a 73-year-old man presenting after 1 month of progressive cough, hemoptysis, fatigue and weight loss. (A) Computed tomography scan of the chest (axial view) showed a large cavitating mass of the posterior right lung apex (arrow) with adjacent posterior chest wall involvement. (B) Magnetic resonance imaging scan of the brain ( $T_{1}$ sequence, axial view) with gadolinium showing a rim-enhancing left cerebellar mass, with 2 small rim-enhancing lesions also seen in the right parietal lobe (not shown). 


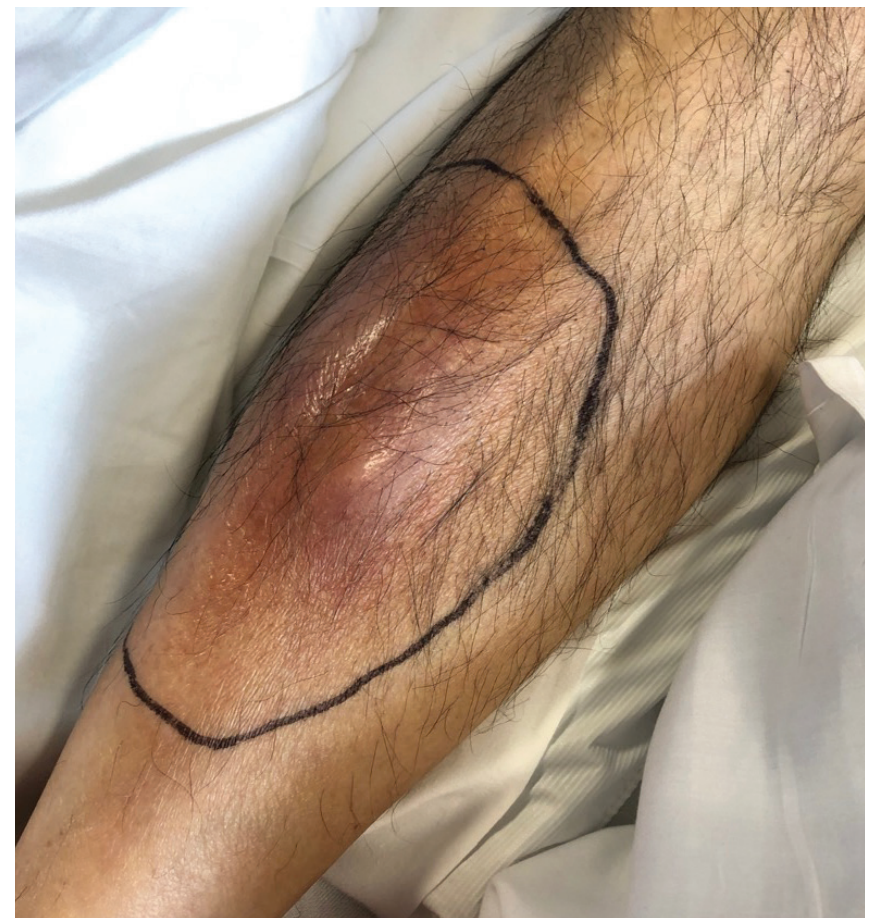

Figure 2: Inspection of the right leg showing a moderately sized area of focal swelling over the anterior tibialis that was tender to palpation, suspicious for a soft-tissue abscess.

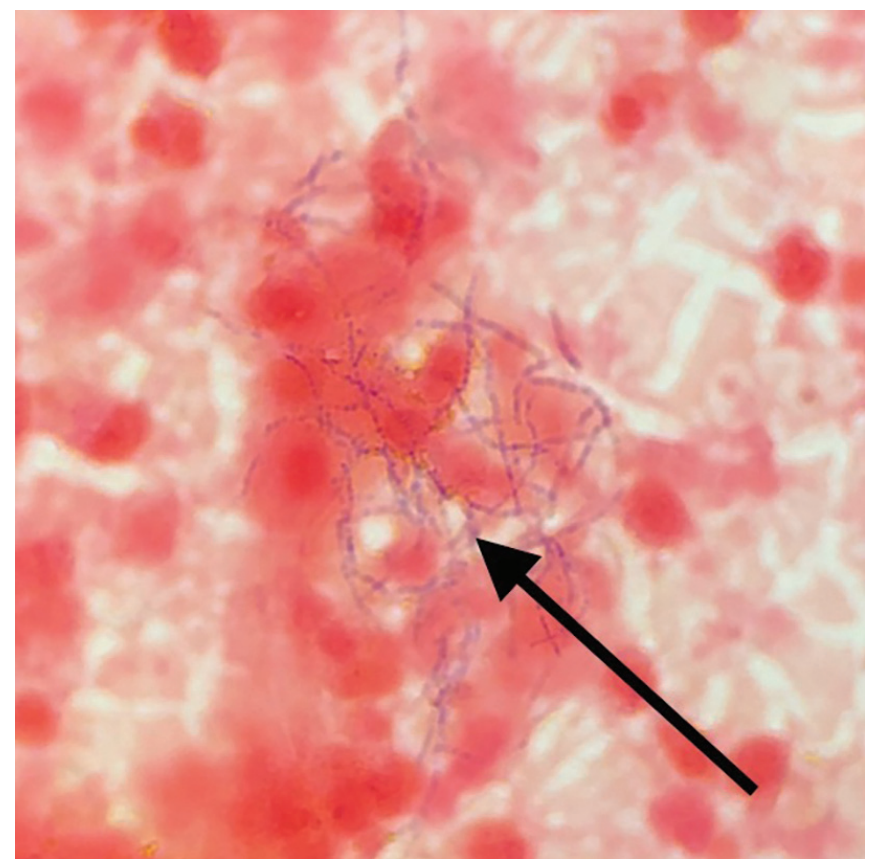

Figure 3: Gram stain of fluid collected from bronchoalveolar lavage showed branching, filamentous gram-positive bacilli, later identified as Nocardia arthritidis (arrow) using matrix-assisted laser desorption ionization time-of-flight (MALDI-TOF) mass spectrometry. Original magnification $\times 1000$.

chest (Figure 1A), which further characterized the mass $(5.4 \mathrm{~cm}$ $\times 4.2 \mathrm{~cm}$ ) in the posterior right lung apex with adjacent chest wall involvement, suspicious for a primary lung malignancy.
This led to an oncology referral. Over the next several weeks, the patient developed right lower-leg swelling, pain and erythema, which made walking difficult. His family physician suspected cellulitis and prescribed a 1-week course of amoxicillin and clavulanic acid, but his symptoms did not improve. At this point, the patient underwent the scheduled brain MRI scan on an outpatient basis; it showed a bulky cerebellar lesion with associated midline shift and substantial vasogenic edema. On seeing the scan, the on-call neurosurgeon recommended an urgent brain biopsy and requested that the patient return immediately to the hospital.

On arrival at the emergency department, the patient was febrile $\left(38.2^{\circ} \mathrm{C}\right)$. His oxygen saturation was $97 \%$, and he had decreased breath sounds in the right upper-lung zone. His right lower leg was focally erythematous, swollen and tender over the anterior tibialis (Figure 2). A neurological examination showed left-sided dysmetria (incoordination). A repeat MRI of the brain with gadolinium showed the original rim-enhancing cerebellar mass, with 2 new small rim-enhancing lesions in the right parietal lobe, suspicious for new intracerebral metastases (Figure 1B).

Given the patient's new onset of fever, we suspected an infectious cause and thought it was prudent to rule out tuberculosis. We advised against a brain biopsy and recommended bronchoscopy. Transbronchial biopsy was negative for malignancy. Gram stain of bronchoalveolar lavage fluid showed partially acid-fast gram-positive branching filamentous bacilli (Figure 3), unlike mycobacteria, which are nonbranching and acid fast. Magnetic resonance imaging of the patient's right leg showed a long intramuscular fluid collection between the lateral tibia and tibialis anterior muscle, which was aspirated, revealing the same organism on gram stain - subsequently identified as Nocardia arthritidis. We diagnosed disseminated nocardiosis and prescribed oral trimethoprim-sulfamethoxazole $15 \mathrm{mg} / \mathrm{kg}$ and IV imipenem $1 \mathrm{~g}$ every 8 hours.

A follow-up MRI 1 month later showed decreased size of the brain lesions. We discharged our patient with a prolonged course of antibiotic therapy, subsequently narrowed to trimethoprimsulfamethoxazole based on susceptibility results (organism was also susceptible to amikacin, ceftriaxone and linezolid, and intermediate to imipenem).

\section{Discussion}

Our patient presented with a cavitary apical lung lesion, a cerebellar brain mass and a lower-leg intramuscular abscess secondary to disseminated nocardiosis. Nocardia species are branching, filamentous gram-positive bacilli found worldwide in soil, dust, water and decaying plant matter. ${ }^{1}$ In the province of Ontario, where our patient resided, 28 endemic strains have been described by the Public Health Laboratory, reflecting the diversity of Nocardia species in the province. ${ }^{2}$ However, infection rates in Canada have not been systematically studied. ${ }^{1}$ People are most commonly infected through inhalation of fragmented cells aerosolized in dusty environments. ${ }^{1,3}$ Other less common routes of entry include ingestion of contaminated 
foods and direct inoculation through the skin after trauma. Given the course of our patient's illness, with respiratory symptoms preceding the development of an intramuscular abscess, inhalation was most likely the route of infection. He frequently gardened at his home, which exposed him to decaying plant matter.

Although as many as one-third of cases may occur in immunocompetent hosts, most reports of Nocardia infections describe immunocompromised hosts with depressed cellmediated immunity. Examples include people with HIV infection, hematologic malignancies (especially lymphoma), hematopoietic stem cell or solid-organ transplant recipients, and those undergoing chemotherapy or taking immunosuppressants such as long-term steroids. ${ }^{1,4}$ Comorbid conditions such as diabetes, chronic obstructive pulmonary disease and alcoholism make people more susceptible. ${ }^{4}$ Our patient's smoking history may have been a predisposing factor.

\section{Diagnosis}

About two-thirds of all primary Nocardia infections occur in the lungs, ${ }^{5}$ and patients most commonly present with a subacute to chronic progressive onset of cough, dyspnea, fever, weight loss and fatigue. ${ }^{5,6}$ However, as many as half of primary pulmonary infections result in disseminated infection. ${ }^{6}$ Most notably, $20 \%$ of all Nocardia infections involve the brain..$^{6,7}$ Hence, patients with confirmed or suspected pulmonary Nocardia infections should have imaging of the brain, as the mortality after a central nervous system (CNS) infection is as high as $50 \% .{ }^{8}$ Furthermore, disseminated nocardiosis can rarely, in $2 \%$ of cases, cause cutaneous manifestations, ${ }^{6-8}$ as was seen in our patient.

A diagnosis of nocardiosis can be difficult to establish, because clinical suspicion of the infection is often required and noncontrast imaging can miss early stages of infection, as was evident in this case. For patients presenting with a lung mass and associated brain lesions, the differential diagnosis must be

Box 1: Differential diagnosis of concurrent lung and brain masses $^{8}$

Malignancy

- Primary lung malignancy, secondary metastatic disease Bacterial infection

- Staphylococcus aureus

- Streptococcus species, especially the Streptococcus anginosus group

- Klebsiella pneumoniae

- Nocardia species

Mycobacterial infection

- Mycobacterium tuberculosis

Fungal infection

- Aspergillus species

- Cryptococcus neoformans

- Mucormycosis broadened from malignancy to include infectious causes such as bacterial, mycobacterial and fungal infections (Box 1). ${ }^{8}$ Hence, all such patients should be isolated, using airborne precautions, because of suspicion of tuberculosis infection, as was the case for our patient. Definitive diagnosis requires obtaining a sample of infected tissue or fluid. ${ }^{8}$ Branching, filamentous gram-positive bacilli indicate the presence of either Nocardia or Actinomyces species. ${ }^{8}$ However, when these organisms are partially acid fast, especially when stained with a modified (Kinyoun) acid-fast stain, this points to a presumptive diagnosis of Nocardia infection, differentiating it from Actinomyces. ${ }^{8}$ Although Nocardia species are difficult and slow to culture, the use of $16 \mathrm{~S}$ rRNA-based polymerase chain reaction and newer techniques, such as matrix assisted laser desorption/ionization time-of-flight (MALDI-TOF) mass spectrometry, are reliable methods for identifying these organisms. ${ }^{1}$

\section{Management}

The first-line treatment for Nocardia infections is trimethoprimsulfamethoxazole, ${ }^{1,2}$ which is active against most species of Nocardia. However, some species, such as N. otitidiscaviarum, $N$. nova and $N$. farcinica, occasionally are resistant. ${ }^{8}$ Although mild disease that is limited to the lungs or skin can be empirically treated with monotherapy, because of the high mortality associated with disseminated or CNS disease, dual coverage is recommended, with the addition of an intravenous agent such as imipenem or amikacin at adequate dosing for CNS penetrance until susceptibilities become available. ${ }^{2,9,10}$ Patients who are immunocompetent and have pulmonary or non-CNS nocardiosis can usually be treated successfully with 6-12 months of therapy. ${ }^{8,10}$ However, patients with disseminated disease involving the CNS, or those who are immunocompromised, require a minimum of 12 months of antibiotic treatment. 8,10 Monitoring for relapse at the end of therapy should be considered, especially in the first year after treatment, when most relapses reportedly occur. ${ }^{11}$ However, there is no authoritative consensus on the optimal duration and method for long-term disease surveillance. Among patients with chronic immunosuppression such as those with advanced HIV, daily trimethoprim-sulfamethoxazole prophylaxis to prevent Pneumocystis pneumonia also decreases the risk of Nocardia recurrence. ${ }^{8}$

\section{Conclusion}

Disseminated nocardiosis is an uncommon but important cause of cavitary lung masses, brain lesions and soft-tissue abscesses. The presence of a lung mass associated with a brain mass can often be mistaken for a metastatic lung malignancy. This patient presented with clinical and radiologic features suggestive of nocardiosis that were initially mistaken for malignancy, putting him at risk of undergoing an invasive surgical procedure (brain biopsy). Hence, the differential diagnosis of this presentation, even in immunocompetent individuals, must include infectious causes, including less common entities such as disseminated Nocardia. 


\section{References}

1. Brown-Elliott BA, Brown JM, Conville PS, et al. Clinical and laboratory features of the Nocardia spp. based on current molecular taxonomy. Clin Microbiol Rev 2006;19:259-82.

2. MCTaggart LR, Doucet J, Witkowska M, et al. Antimicrobial susceptibility among clinical Nocardia species identified by multilocus sequence analysis. Antimicrob Agents Chemother 2015;59:269-75.

3. Saubolle MA, Sussland D. Nocardiosis: review of clinical and laboratory experience. J Clin Microbiol 2003;41:4497-501.

4. Martínez Tomás R, Menéndez Villanueva R, Reyes Calzada S, et al. Pulmonary nocardiosis: risk factors and outcomes. Respirology 2007;12:394-400.

5. Shariff M, Gunasekaran J. Pulmonary nocardiosis: review of cases and an update. Can Respir J 2016;2016:7494202.

6. Lederman ER, Crum NF. A case series and focused review of nocardiosis. Medicine 2004;83:300-13.

7. Beaman BL, Beaman L. Nocardia species: host-parasite relationships. Clin Microbiol Rev 1994;7:213-64.

8. Wilson JW. Nocardiosis: updates and clinical overview. Mayo Clin Proc 2012;87: 403-7.

9. Gombert ME, Aulicino TM. Synergism of imipenem and amikacin in combination with other antibiotics against Nocardia asteroides. Antimicrob Agents Chemother 1983;24:810-1.

10. Zhao P, Zhang X, Du P, et al. Susceptibility profiles of Nocardia spp. to antimicrobial and antituberculotic agents detected by a microplate Alamar Blue assay. Sci Rep 2017;7:43660. doi: 10.1038/srep43660.

11. Anagnostou T, Arvanitis M, Kourkoumpetis TK, et al. Nocardiosis of the central nervous system: experience from a general hospital and review of 84 cases from the literature. Medicine 2014;93:19-32.

\section{Competing interests: None declared.}

This article has been peer reviewed.

The authors have obtained patient consent.

Affiliations: Departments of Medicine (Balachandar, Zhabokritsky, Matukas) and Laboratory Medicine \& Pathobiology (Matukas), University of Toronto; Division of Microbiology (Matukas), St. Michael's Hospital, Unity Health Toronto, Toronto, Ont.

Contributors: All authors contributed substantially to the conception and design of the work. All authors drafted the manuscript, revised it critically for important intellectual content, gave final approval of the version to be published and agreed to be accountable for all aspects of the work.

Correspondence to: Arjun Balachandar, arjun.balachandar@mail.utoronto.ca

The section Cases presents brief case reports that convey clear, practical lessons. Preference is given to common presentations of important rare conditions, and important unusual presentations of common problems. Articles start with a case presentation (500 words maximum), and a discussion of the underlying condition follows (1000 words maximum). Visual elements (e.g., tables of the differential diagnosis, clinical features or diagnostic approach) are encouraged. Consent from patients for publication of their story is a necessity. See information for authors at www.cmaj.ca. 